\title{
11th International Workshop in Developmental Nephrology (IWDN)
}

\author{
Norman D. Rosenblum
}

Received: 4 May 2011 / Accepted: 4 May 2011 / Published online: 24 May 2011

(C) IPNA 2011

This special issue of Pediatric Nephrology is devoted to manuscripts that demonstrate cutting-edge research in the field of developmental nephrology. Each of the 25 peerreviewed papers were submitted by participants in the 11 th International Workshop in Developmental Nephrology (IWDN), held at the Mohonk Mountain House, New Paltz, New York, USA, 24-27 August, 2010. The 11th IWDN was sponsored by the International Pediatric Nephrology Association (IPNA) in coordination with the 2010 IPNA Congress and was supported, as well, by grants awarded by the National Institutes of Health USA and the March of Dimes. More than 100 established and new investigators in the fields of pediatric nephrology, developmental biology and physiology, genetics, and cell biology gathered to address new knowledge in the field of developmental kidney disorders.

I was privileged to chair the 11th IWDN with the support of Jordan Kreidberg, co-chair, and an international expert organizing committee constituted by Anita Aperia, Carlton Bates, John Bertram, Robert Chevalier, Gregory Dressler, Iain Drummond, Samir El-Dahr, Frederick Kaskel, Paul Goodyer, Lisa Guay-Woodford, Melissa Little, Cathy Mendelsohn, Ryuichi Nishinakamura, Remi Salomon, Isidro Salusky, and Lisa Satlin, and with expert administrative support by Juliet Rodrigo and the staff of the Mohonk Mountain House. The 11th IWDN greatly benefited from the close coordination between the organizing committee and Dr. Frederick Kaskel, president of the 2010 IPNA Congress.

N. D. Rosenblum $(\bowtie)$

The Hospital for Sick Children and the University of Toronto, Toronto, ON, Canada

e-mail: norman.rosenblum@sickkids.ca
This collection of manuscripts reflects the overall theme of the 11th IWDN: "Genetic Programming and the Kidney: Progenitors, Signaling, and Morphogenesis in Health and Disease." This overall theme was elaborated by the organization of the IWDN into keynote lectures and six subthemes articulated by 46 oral presentations and 32 poster presentations. The manuscript by Christensen and Nedergaard captures the content of one of the keynote lectures delivered by Majken Nedergaard, University of Rochester Medical Center, Rochester, NY, USA, on structural and functional imaging of live cells. Therein lies a cross-cutting theme within the IWDN, namely, to highlight progress in nonrenal developmental systems that can be beneficial to kidney researchers. All other manuscripts in this special issue relate to one of the six subthemes: "Stem Cells, Progenitors, and Regeneration" (manuscripts by Yeagy and Cherqui, Davidson, PodeShakked and Dekel, Hendry et al., and Nishinakamura), "Molecular Mechanisms and Patterning of the Metanephric Field" (manuscripts by Dressler, Oxburgh et al., Potter et al., Wellik, and Wessely and Tran), "Morphogenesis of the Renal Collecting System" (manuscripts by Bates, Reidy and Tufro, Yosypiv, and Yu), "Signaling and Control of Nephron Formation" (manuscripts by Bertram et al., and Cain and Rosenblum), "Patterning and Morphogenesis of the Lower Urinary Tract" (manuscripts by Stewart and Bouchard, Chevalier et al., Murawski et al., and Ingraham and McHugh), and "Molecular Signaling and Epithelial Differentiation" (manuscripts by Khodus et al., Rosenberg et al., Miner, and Hermle et al.). Readers of this special issue may find it useful to group manuscripts according to these subthemes to derive particular benefit from their reading.

The discipline of developmental nephrology has undergone remarkable transitions since the 1 st IWDN. Thirty 
years ago, this discipline was focused on whole-organ physiology and glomerular and tubular functions. The knowledge gained from these studies informed the clinical approach to neonatal fluid and electrolyte homeostasis and renal dysfunction in infants and young children. Integrative physiology was overtaken by the "revolution" in molecular biology and developmental genetics. This revolution ushered in an era of structural and functional studies of single molecules and the molecular control of cellular functions, cell differentiation, and morphogenesis.

The manuscripts contained in this special edition demonstrate that the "revolution" in biology continues. The synergistic tools of genetics and developmental biology are being used with increasing sophistication and specificity to identify the individual and combined contributions of genes that instruct the differentiation and morphogenesis of cells and cellular structures during renal morphogenesis. These advances are highlighted in papers within the subthemes "Stem Cells, Progenitors and Regeneration," "Molecular Mechanisms and Patterning of the Metanephric Field," "Morphogenesis of the Renal Collecting System," and "Signaling and Control of Nephron Formation."

After a prolonged period of investigation during which some wondered whether the reductionism of molecular biology would inform human developmental nephrology, it is now clear that the knowledge accumulated is beginning to inform several issues central to human renal development and developmental dysfunction. I highlight four examples of progress directly related to human physiology and pathophysiology and discussed within this special edition. First, Bertram et al. highlight the importance of nephron number to renal function, not only in infants and children, but also in adults. We have reached a stage of scientific knowledge in which many molecular mechanisms that control nephron number have been identified. The genes that control these mechanisms have been linked to human diseases characterized by variable degrees of nephron deficiency. An increasing number of studies are dissecting the genetic contribution to renal hypoplasia; studies that have identified the genetic control of renal formation are providing a basis on which the functional contribution of these genes may be understood. Second, it is increasingly clear that renal developmental abnormalities are likely to arise from epigenetic mechanisms that arise during pregnancy related to malnutrition and stress, examples of what are likely to be only a subset of a large number of causative unhealthy states during pregnancy. Here, Rosenberg et al. summarize emerging knowledge of mechanisms by which gene expression is modified during development via epigenetic modifications. Third, identification of stem cells and progenitors in developing tissues and in the kidney, specifically, is providing a foundation on which to develop exciting new strategies aimed at correcting monogenic defects that target kidney function (see paper by Yeagy and Cherqui). Fourth, molecules involved in solute transport are now being implicated in developmental functions. Khodes et al. highlight a calcium-dependent signaling pathway downstream of the sodium-potassium-adenosine triphosphatase (Na-K-ATPase) that protects the embryonic kidney from the adverse effects of malnutrition. Hermle et al. provide evidence that proton pumps, traditionally associated with acid-base regulation, are implicated in growthfactor-dependent control of epithelial morphogenesis. These examples illustrate that we are in the midst of an increasingly integrative phase of research in which observations at the level of single cells and threedimensional processes can be related to organ function, susceptibility, and response to injury and lifelong capacity.

Finally, I thank Dr. Michel Baum, editor, Pediatric Nephrology, for his constant support in making this special edition of the journal possible. 